\title{
INTEGRAL detection of hard X-rays from NGC 6334: nonthermal emission from colliding winds or an AGN?`
}

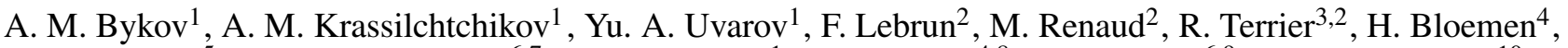 \\ B. McBreen ${ }^{5}$, T. J.-L. Courvoisier ${ }^{6,7}$, M. Yu. Gustov ${ }^{1}$, W. Hermsen ${ }^{4,8}$, J.-C. Leyder ${ }^{6,9}$, T. A. Lozinskaya ${ }^{10}$, \\ G. Rauw ${ }^{9}$, and J.-P. Swings ${ }^{9}$
}

1 A. F. Ioffe Institute for Physics and Technology, 26 Polytechnicheskaia, 194021 St. Petersburg, Russia e-mail: byk@astro.ioffe.ru

2 CEA-Saclay, DSM/DAPNIA/Service d'Astrophysique, 91191 Gif-sur-Yvette Cedex, France

3 APC-UMR 7164, 11 place M. Berthelot, 75231 Paris, France

${ }^{4}$ SRON Netherlands Institute for Space Research, Sorbonnelaan 2, 3584 CA Utrecht, The Netherlands

5 Department of Physics, University College Dublin, Dublin 4, Ireland

6 INTEGRAL Science Data Centre, Chemin d'Écogia 16, 1290 Versoix, Switzerland

7 Geneva Observatory, Chemin des Maillettes 51, 1290 Sauverny, Switzerland

8 Astronomical Institute "Anton Pannekoek", University of Amsterdam, Kruislaan 403, 1098 SJ Amsterdam, The Netherlands

9 Institut d'Astrophysique et de Géophysique, Université de Liège, Allée du 6 Août 17, Bât. B5c, 4000 Liège, Belgium

10 Sternberg Astronomical Institute, Moscow State University, 13 Universitetskij, 119899 Moscow, Russia

Received 1 September 2005 / Accepted 20 December 2005

ABSTRACT

Aims. We report the detection of hard X-ray emission from the field of the star-forming region NGC 6334 with the International Gamma-Ray Astrophysics Laboratory INTEGRAL.

Methods. The JEM-X monitor and ISGRI imager aboard INTEGRAL and Chandra ACIS imager were used to construct 3-80 keV images and spectra of NGC 6334.

Results. The 3-10 keV and 10-35 keV images made with JEM-X show a complex structure of extended emission from NGC 6334. The ISGRI source detected in the energy ranges $20-40 \mathrm{keV}, 40-80 \mathrm{keV}$, and 20-60 keV coincides with the NGC 6334 ridge. The 20-60 keV flux from the source is $(1.8 \pm 0.37) \times 10^{-11} \mathrm{erg} \mathrm{cm}^{-2} \mathrm{~s}^{-1}$. Spectral analysis of the source revealed a hard power-law component with a photon index about 1 . The observed X-ray fluxes are in agreement with extrapolations of X-ray imaging observations of NGC 6334 by Chandra ACIS and ASCA GIS. Conclusions. The X-ray data are consistent with two very different physical models. A probable scenario is emission from a heavily absorbed, compact and hard Chandra source that is associated with the AGN candidate radio source NGC 6334B. Another possible model is the extended Chandra source of nonthermal emission from NGC 6334 that can also account for the hard X-ray emission observed by INTEGRAL. The origin of the emission in this scenario is due to electron acceleration in energetic outflows from massive early type stars. The possibility of emission from a young supernova remnant, as suggested by earlier infrared observations of NGC 6334, is constrained by the non-detection of ${ }^{44} \mathrm{Ti}$ lines.

Key words. gamma rays: observations - X-rays: ISM - star forming regions - individual: NGC 6334

\section{Introduction}

NGC 6334 is a star forming (SF) complex of a total bolometric luminosity $\sim 1.9 \times 10^{6} L_{\odot}$ associated with a giant molecular cloud of mass $\sim 1.6 \times 10^{5} M_{\odot}$ (e.g. Loughran et al. 1986). The complex structure of NGC 6334 with a number of clearly

* The present work is partly based on observations with INTEGRAL, an ESA project with instruments and a science data centre funded by ESA member states (especially the PI countries: Denmark, France, Germany, Italy, Switzerland, Spain), Czech Republic and Poland, and with the participation of Russia and the USA. This research has made use of data obtained from the NASA High Energy Astrophysics Science Archive Research Center. separated and localized SF sites was established by radio and infrared (IR) observations. Massive SF sites reside along a ridge of size about $20^{\prime} \times 3^{\prime}(\sim 10 \times 1.5 \mathrm{pc})$ and are associated with the peaks in the main ridge of molecular gas. The ridge can be seen in many energy bands ranging from $843 \mathrm{MHz}$ radio to several $\mathrm{keV} \mathrm{X}$-rays. The standard tracers of SF regions such as [C II] $158 \mu \mathrm{m}$, [O I] $145 \mu \mathrm{m}$, and [O I] $63 \mu \mathrm{m}$, as well as rich molecular emission spectra revealed structured emission of NGC 6334 with clumps of far-infrared (FIR) sources. Recently Kraemer \& Jackson (1999) reviewed molecular gas in NGC 6334 and constructed detailed maps of the CO, CS, and $\mathrm{NH}_{3}$ emission regions. They found a complex structure of the gas distribution with a number of molecular filaments 
and bubbles. High resolution VLA observations of NGC 6334 by Carral et al. (2002) revealed shell-like structures of diameters from 0.12 to $3.5 \mathrm{pc}$ probably tracing stellar winds. Nonthermal radio emission features, $\mathrm{H}_{2} \mathrm{O}, \mathrm{OH}$, and methanol maser sources, as well as high magnetic fields (about $200 \mu \mathrm{G}$ ) were observed in NGC 6334 (e.g. Sarma et al. 2000, and references therein). We adopt the distance of $\sim 1.7 \mathrm{kpc}$ to NGC 6334 (Neckel 1978).

McBreen et al. (1979) discovered a bright $\left(\sim 1.9 \times 10^{5} L_{\odot}\right.$ bolometric luminosity) source of IR emission in the southwestern part of the SF ridge which was not detected in previous IR observations of the region. The source position is associated with $\mathrm{H}_{2} \mathrm{O}$ and $\mathrm{OH}$ masers. Bipolar structure of extended $\mathrm{H}_{2}$ emission and an energetic outflow were found in the source (as well as in a nearby FIR source). The nature of the apparent variability was attributed by McBreen et al. (1979) to a sudden luminosity onset during early SF phenomena or, alternatively, to a supernova (SN) hidden in a molecular cloud. Young SN remnants (SNRs) possibly hidden in dense molecular clouds can be identified by their gamma-ray emission lines from ${ }^{44} \mathrm{Ti}$ with the ISGRI detector (Lebrun et al. 2003). Hard $\mathrm{X}$-ray observations are a perfect tool to look for a SNR hidden in a dense molecular cloud. Being compact sites of powerful kinetic energy release and fast shock waves generated by stellar winds and SNRs, massive SF regions are expected to have nonthermal emission components (e.g. Bykov 2001). This possibility strongly motivates INTEGRAL data analysis of NGC 6334.

NGC 6334 was observed with ASCA (Matsuzaki et al. 1999; Sekimoto et al. 2000). X-ray emission was detected from the SF ridge and from the source AXJ 1720.3-3544 that is about $10^{\prime}$ to the north of the center of the ridge. The emission spectrum of the ridge sources was fitted with a thermal hot plasma of temperature $\sim 9 \mathrm{keV}$. The source AXJ 1720.3-3544 was identified as the B0.5e star CD-35 11482. Recently Ezoe et al. (2005) analyzed Chandra data on NGC 6334. In addition to 800 point sources in the field they found a diffuse $\mathrm{X}$-ray emission region of $5 \times 9 \mathrm{pc}$ size and of luminosity $2 \times 10^{33} \mathrm{erg} \mathrm{s}^{-1}$. The authors suggested that thermal emission of several $\mathrm{keV}$ is due to plasma heated by stellar wind shocks, while a flat continuum is due to accelerated particles.

However, it is not easy to distinguish hot thermal gas from nonthermal emission generated by accelerated particles with an energy band that is limited to $10 \mathrm{keV}$. We present below the first hard X-ray (3-80 keV) images and spectra of NGC 6334 made with INTEGRAL.

\section{Observations and data analysis}

\subsection{INTEGRAL observations}

The regions surrounding NGC 6334 have been observed with the ISGRI imager (Ubertini et al. 2003; Lebrun et al. 2003) and JEM-X monitor (Lund et al. 2003) aboard INTEGRAL (Winkler et al. 2003).

We analysed $420 \mathrm{ks}$ of fully coded field of view (FCFOV) data from ISGRI and $92 \mathrm{ks}$ of FCFOV data from $J E M-X$. The data were taken during revolutions 46-167 (28 Feb. 2003-27 Feb. 2004) and are now public.
The INTEGRAL data have been reduced with the standard off-line scientific analysis OSA 5.0 version (Courvoisier et al. 2003). The standard good time selection criteria were applied; only science windows with more than $100 \mathrm{~s}$ of good time were considered.

The INTEGRAL images of the field around NGC 6334 reveal the complex morphology of the X-ray emission. Archival ROSAT, ASCA, Chandra and CGRO EGRET observations of NGC 6334 were analyzed in addition to the INTEGRAL data. Available archive MOST, VLA, IRAS, MSX, 2MASS, and DSS data was used to construct a multiwavelength picture of a few degrees in size around the NGC 6334 complex.

Hard X-ray emission from NGC 6334 can be efficiently studied by combining ISGRI and high resolution Chandra observations. We used two Chandra $40 \mathrm{ks}$ observations (ObsIDs 2573 and 2574) of NGC 6334 performed on 31 Aug. and 2 Sep. 2002 (P. I. Y. Ezoe). The source was imaged with four ACIS-I CCDs. The event files were processed with the standard CIAO tools (ver. 3.1, CALDB 2.28).

In Fig. 1 we show multiwavelength images of NGC 6334. The NGC 6334 ridge, as seen in IR, is shown on panel d, while its counterparts at $843 \mathrm{MHz}$ and $1.4 \mathrm{GHz}$ radio and in $\mathrm{keV}$ $\mathrm{X}$-rays are visible on panels $\mathrm{a}, \mathrm{b}$ and $\mathrm{c}$. The morphology of the $10-35 \mathrm{keV} J E M-X$ image is very similar to that of $3-10 \mathrm{keV}$; the $20-40 \mathrm{keV}$ and $40-80 \mathrm{keV}$ maps by ISGRI are also very similar to the 20-60 keV map shown in Fig. 1d. The apparent $J E M-X$ NGC 6334B counterpart is $\sim 2.5^{\prime}$ from the Chandra position (within the JEM-X resolution of $3^{\prime}$ ).

The ROSAT RASS map shows a patchy structure caused by the distribution of X-ray sources and strong local $N_{\mathrm{H}}$ variations in the molecular cloud complex. The $7-8 \mathrm{keV}$ map of Chandra consists of extended emission regions correlated with the $1.4 \mathrm{GHz}$ radio excesses.

From cross calibration with the Crab we derived the following flux estimates for the ISGRI source: $(1.8 \pm$ $0.37) \times 10^{-11} \mathrm{erg} \mathrm{cm}^{-2} \mathrm{~s}^{-1}$ in the $20-60 \mathrm{keV}$ band, $(1.1 \pm$ $0.26) \times 10^{-11} \mathrm{erg} \mathrm{cm}^{-2} \mathrm{~s}^{-1}$ in the $20-40 \mathrm{keV}$ band, and $(1.2 \pm 0.39) \times 10^{-11} \mathrm{erg} \mathrm{cm}^{-2} \mathrm{~s}^{-1}$ in the $40-80 \mathrm{keV}$ band. The significances of the detections are $4.9,4.2,3.1 \sigma$, respectively.

\subsection{Spectral analysis of the hard $X$-ray emission}

The modest resolution of the INTEGRAL high energy images (panels $\mathrm{c}$ and $\mathrm{d}$ in Fig. 1) strongly motivates a joint analysis of high resolution Chandra observations with that of INTEGRAL to constrain the nature of the nonthermal emission. We made a spectral analysis of the Chandra $0.8-8.0 \mathrm{keV}$ emission extracted from the rectangular ISGRI excess region of size $5^{\prime} \times 10^{\prime}$, shown in Figs. 1a, b and c.

Inspection of the Chandra sources in the field revealed two probable scenarios. We find that the observed ISGRI flux may come from a bright point-like hard Chandra source located at (FK5: 17:20:21.8; -35:52:48) that is clearly seen in Fig. 1b. The source coincides with the known radio source NGC 6334B (FK5: 17:20:21.8; -35:52:48.2) studied by Moran and collaborators (see Moran et al. 1990, and references therein). 


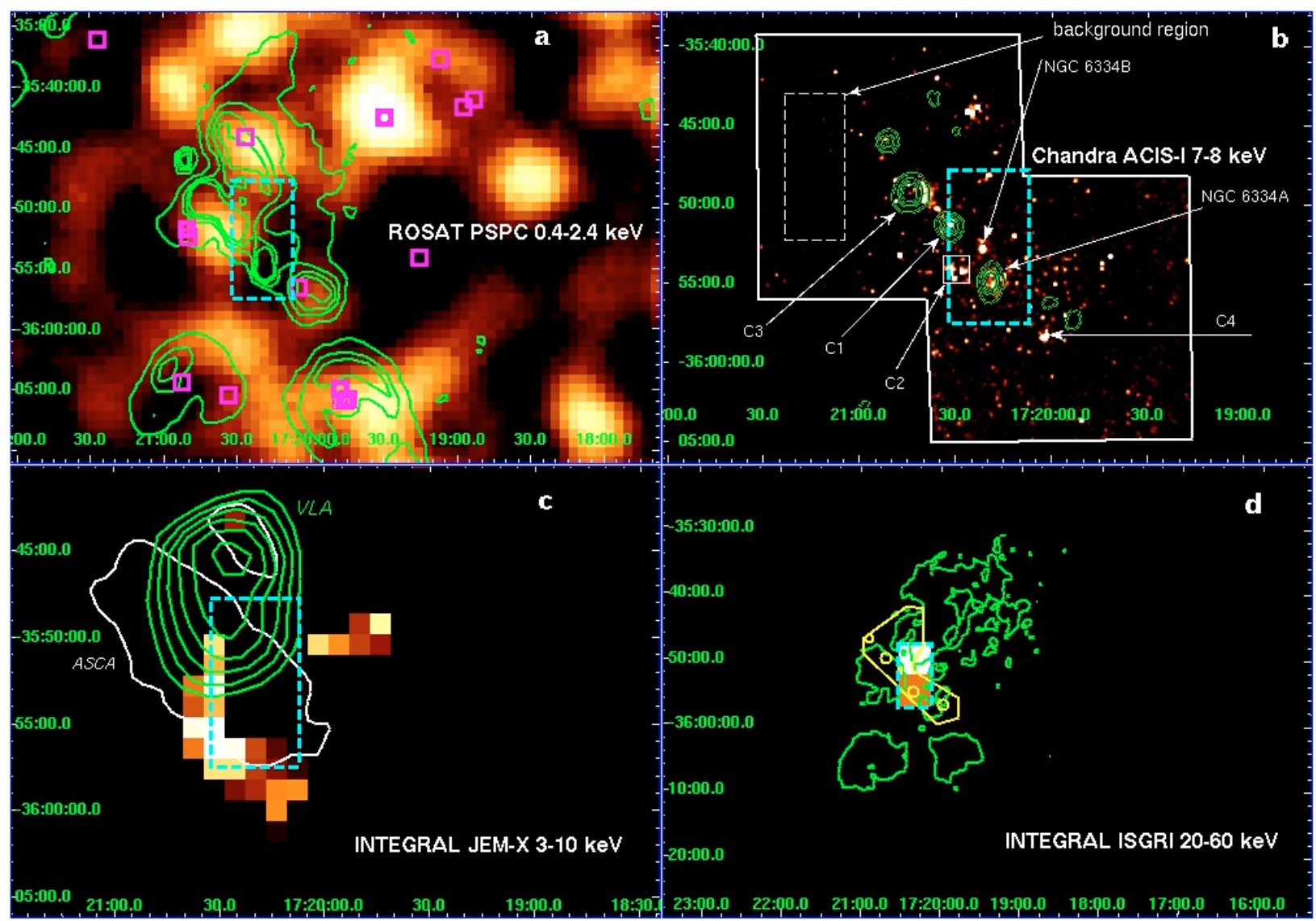

Fig. 1. a) ROSAT RASS 0.4-2.4 keV map with MOST MGPS $843 \mathrm{MHz}$ contours. Massive stars, visible in the optical band are shown by boxcircles (O stars) and boxes (B stars). b) Chandra 7-8 keV map smoothed with a 2 pixel Gaussian kernel and VLA NVSS $1.4 \mathrm{GHz}$ contours. The background region used for Chandra analysis is marked as well as the main sources of Chandra emission within the ISGRI excess. c) JEM-X 3-10 keV map with ASCA GIS 6-10 keV contours and VLA GPS $8.35 \mathrm{GHz}$ contours. d) ISGRI 20-60 keV map with DSS-R contours. The P-shaped region with circles indicates the NGC 6334 ridge with SF complexes as seen in $71 \mu \mathrm{m}$ IR band by Loughran et al. (1986). Bright ISGRI pixels are shown on panels a)-c) as dashed rectangles.

They suggested that NGC 6334B is a background extragalactic source.

Alternatively, the ISGRI hard emission may originate from the extended rectangular region covered by the two bright ISGRI pixels, with the emission within a radius of $10^{\prime \prime}$ from NGC 6334B subtracted. We will now refer to it as the hard extended emission (HEE) region. The HEE region is not dominated by one or two bright point-like Chandra sources, but it has a structured morphology with a few bright clumps. The clumps may be either extended or consist of many weak sources. In the HEE region we further selected three extended regions associated with the X-ray emission clumps. They are marked as NGC 6334A, C1, and C2 in Fig. 1b. The sources NGC 6334A and C1 are bounded by an NVSS radio contours shown in Fig. 1b. NGC 6334A contains a radio shell of $15^{\prime \prime}$ diameter and of about $10 \mathrm{Jy}$ flux density at $6 \mathrm{~cm}$ found by Carral et al. (2002). The HII region NGC 6334A is coincident with a bright IR source (McBreen et al. 1979). In Fig. 4 we present a zoomed Chandraimage of the region. We found no clear Chandra counterpart to the central source of the NGC 6334A radio shell. The central source of $6.1 \pm 0.9$ mJy flux density at $3.5 \mathrm{~cm}$ was found by Carral et al. (2002) in high resolution VLA data. However, there is a possible Chandra counterpart
Table 1. Parameters of $0.8-8.0 \mathrm{keV}$ Chandra fits for sources within the HEE region shown in Fig. $1 \mathrm{~b}$.

\begin{tabular}{c|cccc}
\hline \hline Source & NGC 6334B & NGC 6334A & C1 & C2 \\
\hline$N_{\mathrm{H}}{ }^{a}$ & $13 \pm 3.0$ & $2.7 \pm 0.5$ & $0.6 \pm 0.1$ & $0.5 \pm 0.1$ \\
$\Gamma$ & $0.9 \pm 0.5$ & $1.4 \pm 0.3$ & $1.7 \pm 0.1$ & $1.5 \pm 0.1$ \\
$\chi_{v}^{2} /$ d.o.f. & $19 / 47$ & $30 / 51$ & $99 / 92$ & $48 / 62$ \\
$F_{\mathrm{x}}{ }^{b}$ & 2.9 & 1.0 & 0.9 & 0.6 \\
\hline
\end{tabular}

${ }^{a}$ Hydrogen column density, in $10^{22} \mathrm{~cm}^{-2}$.

${ }^{b}$ Unabsorbed flux in 2.0-7.0 keV band, in $10^{-12} \mathrm{erg} \mathrm{cm}^{-2} \mathrm{~s}^{-1}$.

to the IRS 19 source also present within the radio shell (see Fig. 4).

For Chandra spectral analysis we used only the northern Chandra pointing (ObsID 2574), because the other one is contaminated by flares. The background region used is shown in Fig. 1b. We also used blank-sky background models provided by the CIAO. The results of Chandra spectral analysis of the selected sources within the HEE region are summarized in Table 1. The Chandra spectra of the most prominent regions NGC 6334B and NGC 6334A are shown in Fig. 2. 

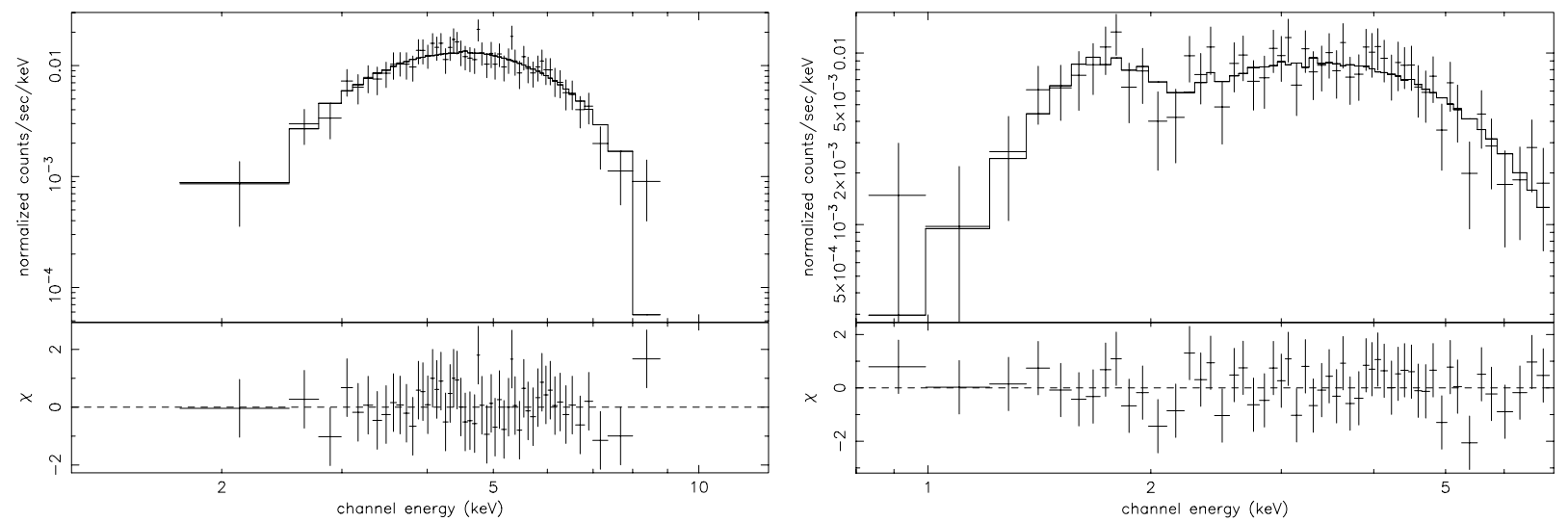

Fig. 2. Chandra spectra of NGC 6334B (left panel) and of NGC 6334A (right panel). Fits are shown as solid lines and have $\chi_{v}^{2} \lesssim 1$.
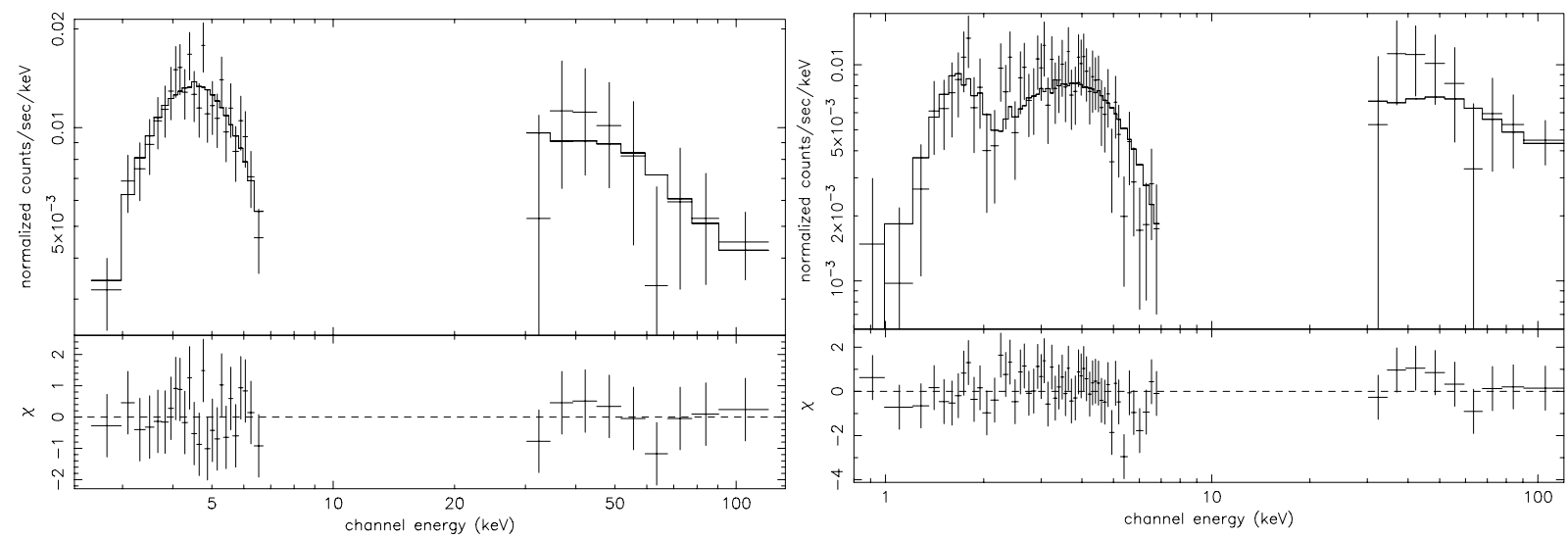

Fig. 3. The combined Chandra-ISGRI spectra of NGC 6334B (left panel) and of NGC 6334A (right panel). Fits are shown as solid lines and have $\chi_{v}^{2} \lesssim 0.75$.

The combined Chandra-ISGRI spectra of these two sources are shown in Fig. 3.

The combined Chandra-ISGRI data for NGC 6334B are fitted with $\chi_{\nu}^{2}=0.52$ by a power-law with $N_{\mathrm{H}}=(1.2 \pm 0.1) \times$ $10^{23} \mathrm{~cm}^{-2}$, photon index $\Gamma=0.9 \pm 0.1$ and normalization $K=(2.9 \pm 0.9) \times 10^{-4} \mathrm{ph} \mathrm{cm}^{-2} \mathrm{~s}^{-1} \mathrm{keV}^{-1}$ at $1 \mathrm{keV}$. The combined Chandra-ISGRI data for NGC 6334A are fitted with $\chi_{v}^{2}=0.75$ by a power-law with $N_{\mathrm{H}}=(1.5 \pm 0.2) \times 10^{22} \mathrm{~cm}^{-2}$, photon index $\Gamma=0.5 \pm 0.1$ and normalization $K=(6.1 \pm 1.4) \times$ $10^{-5} \mathrm{ph} \mathrm{cm}^{-2} \mathrm{~s}^{-1} \mathrm{keV}^{-1}$ at $1 \mathrm{keV}$.

The Chandra spectrum of the whole HEE region is shown in Fig. 5. The spectrum contains an iron line feature at (6.5 \pm $0.1) \mathrm{keV}$. The Chandra data are consistent with the ASCA spectrum of the combined FIR cores I-V reported by Sekimoto et al. (2002) to be fitted by a Raymond-Smith temperature $k T=9.0_{-1.8}^{+3.3} \mathrm{keV}$ and a line at $(6.6 \pm 0.1) \mathrm{keV}$. Alternatively, the Chandra spectrum of the HEE region can be modeled by a power-law with the photon index $\Gamma=1.5 \pm 0.1$, and $N_{\mathrm{H}}=(1.3 \pm 0.2) \times 10^{22} \mathrm{~cm}^{-2}$. In the latter case, the iron line has a non-thermal origin. The $2-7 \mathrm{keV}$ unabsorbed flux of the HEE region is $4.4 \times 10^{-12} \mathrm{erg} \mathrm{cm}^{-2} \mathrm{~s}^{-1}$. Analysis of the Chandra spectra of the extended HEE region above $7 \mathrm{keV}$ depends strongly on the applied background model due to substantial spatial variations of Chandra background above $7 \mathrm{keV}$. The background obtained from regions of different sizes and shapes was applied to the spectrum of the HEE region and yielded different values for the countrate in the $7-10 \mathrm{keV}$ energy bin. The $7-10 \mathrm{keV}$ rate plotted in Fig. 5 was obtained for the blank sky ${ }^{1}$ background and thus should be regarded as an upper limit. The limit is compatible with the hypothesis that the origin of a substantial part of the $20-60 \mathrm{keV}$ emission from NGC 6334 is from the HEE region. The Chandra spectrum of the point-like source NGC 6334B extends up to $9 \mathrm{keV}$ (see Fig. 2 left panel) and is also well matched to the observed ISGRI flux. Thus, more observations (e.g. with XMM-Newton) are required to get a real constraint on the relative contribution of the HEE region and the AGN-like NGC 6334B source to the ISGRI excess.

The five FIR core sources along the ridge of emission in NGC 6334 were detected in the Chandra images and have hard emission components above $5 \mathrm{keV}$. To estimate their relative contribution to the hard X-ray emission of NGC 6334 we compare their fluxes in the $5-7 \mathrm{keV}$ band where the detection is still significant. More than a half of the hard X-ray emission of the whole NGC 6334 ridge (unabsorbed flux $\sim 2 \times 10^{-12} \mathrm{erg} \mathrm{cm}^{-2} \mathrm{~s}^{-1}$ in the 5-7 keV regime) comes from the point-like source NGC 6334B and the HEE region. FIR cores II and V (indicated as C3 and C4 in Fig. 1b) are situated just a few arcminutes outside the HEE region and

1 fttp://cda.harvard.edu/pub/arcftp/caldb/ 
contribute about $\left(\sim 0.5 \times 10^{-12} \mathrm{erg} \mathrm{cm}^{-2} \mathrm{~s}^{-1}\right)$ and $(\sim 0.3 \times$ $10^{-12} \mathrm{erg} \mathrm{cm}^{-2} \mathrm{~s}^{-1}$ ), respectively, to the $5-7 \mathrm{keV}$ emission of the whole ridge. Given the proximity of the sources $\mathrm{C} 3$ and $\mathrm{C} 4$ to the HEE region it is not possible with the angular resolution of the ISGRI camera to make a meaningful conclusion on the actual contribution of the FIR cores II and V to the observed hard emission above $20 \mathrm{keV}$.

We have analysed the archival CGRO-EGRET data and derived the following upper limits for gamma-ray emission from NGC 6334: $F<9.9 \times 10^{-8} \mathrm{ph} \mathrm{cm}^{-2} \mathrm{~s}^{-1}$ for $E>100 \mathrm{MeV}$, and $F<1.2 \times 10^{-8} \mathrm{ph} \mathrm{cm}^{-2} \mathrm{~s}^{-1}$ for $E>1000 \mathrm{MeV}$. These limits imply a break in the spectrum of NGC 6334 at some energy above $100 \mathrm{keV}$.

\section{Discussion}

From the spectral analysis of IBIS-ISGRI data we can conclude that the hard X-ray emission has a nonthermal nature. The X-ray data are consistent with two different physical models, or with their combination. A plausible scenario is emission from a heavily absorbed, compact and hard Chandra source that is probably associated with the obscured accreting source NGC 6334B. An alternative model is emission from the hard extended Chandra sources related to NGC 6334A and some other extended structures in the NGC 6334 SFR. The origin of the emission in this scenario is due to electron acceleration in energetic outflows from massive early type stars.

\subsection{An obscured AGN source}

A possible interpretation of the observed hard X-rays could be an obscured AGN. The Chandra source position coincides with better than $1^{\prime \prime}$ accuracy with the NGC 6334B radio source studied by Moran et al. (1990). The HI observations of NGC 6334B revealed a prominent feature with large negative velocity at about $100 \mathrm{~km} \mathrm{~s}^{-1}$ indicating that the distance to the source must be at least $6 \mathrm{kpc}$. Moran et al. (1990) suggested that NGC 6334B is unrelated to the NGC 6334 molecular cloud complex and is likely to be extragalactic. They also summarized measurements of the $\lambda=6 \mathrm{~cm}$ flux variability with the flux variation from $0.18 \mathrm{Jy}$ on Jun. 19,1978 to $0.79 \mathrm{Jy}$ on Feb. 20, 1981 with the measured fluxes above $0.4 \mathrm{Jy}$ till Jul. 1988. We searched for ISGRI flux variations in the bright $5^{\prime}$ pixel at the NGC 6334B position. Given the limited count statistics of the ISGRI source we grouped events within three time intervals to make the light curve presented in Fig. 6. No significant flux variations in $20-40 \mathrm{keV}$ band on a year timescale were found. The data allows to exclude flux variations above a factor $>3$ on the year time scale. We also made a light curve for Chandra $0.8-8.0 \mathrm{keV}$ flux for NGC 6334B presented in Fig. 7. No significant flux variations on the hour time scale were found.

Simultaneous radio and X-ray observations can be used to distinguish between two different types of obscured galactic sources (see e.g. Fender 2005, for a review). Radio fluxes of galactic black hole binaries in the low-hard states with an X-ray flux $\lesssim 10 \mathrm{mCrab}(2-10 \mathrm{keV})$ are below $10 \mathrm{mJy}$. The radio fluxes of $\gtrsim 100 \mathrm{mJy}$ level were detected only in the high-soft states

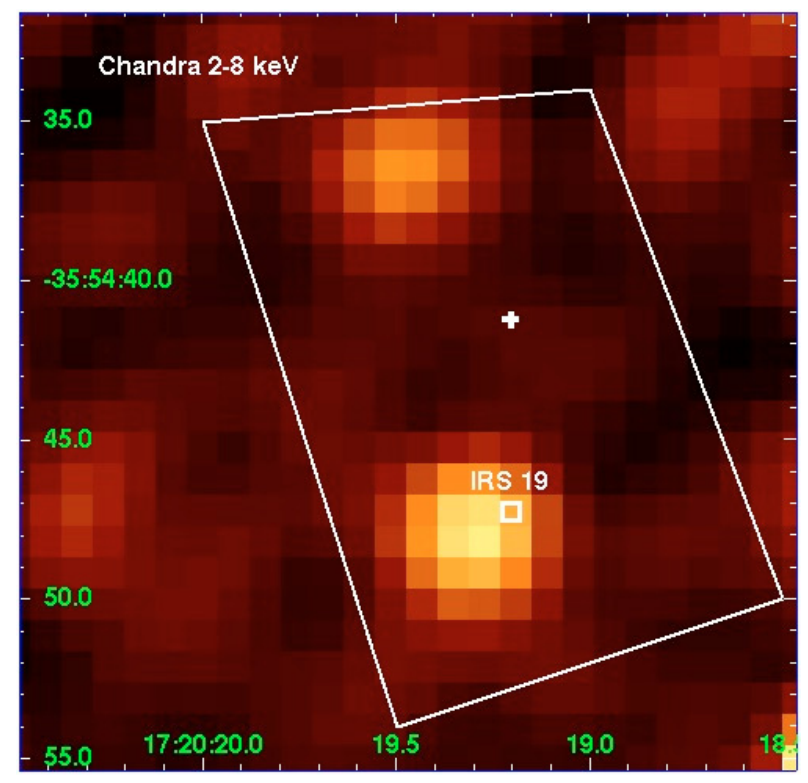

Fig. 4. Chandra 2.0-8.0 keV image of NGC 6334A. Position of the central point VLA source (taken from Carral et al. 2002) is marked by a cross, while that for the IRS 19 is marked by a box, the boundary of the VLA radio shell is indicated as a parallelogram-like frame.

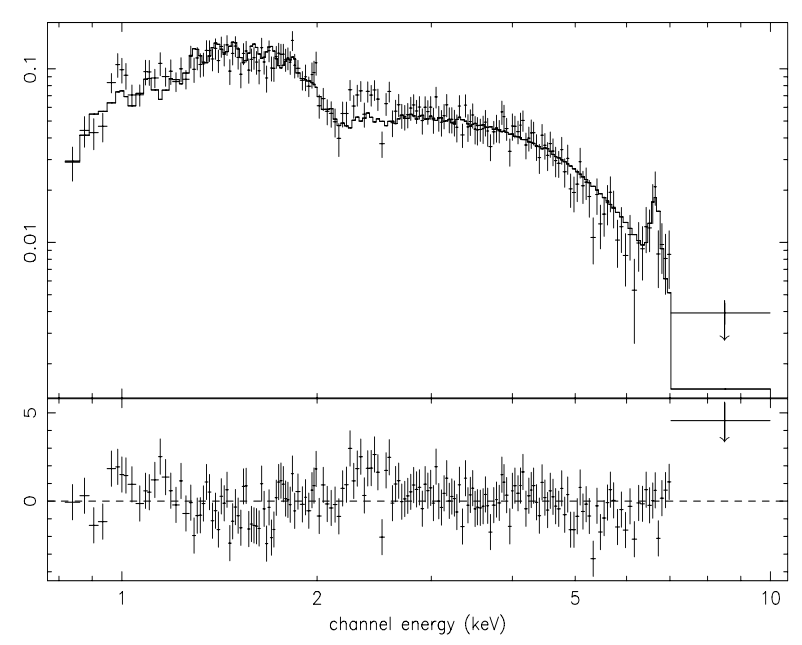

Fig. 5. Chandra spectrum of the HEE region.

with $2-10 \mathrm{keV}$ X-ray flux $\sim 1 \mathrm{Crab}$. The radio fluxes of some observed X-ray binaries with neutron stars are only below that with black holes (e.g. Muno et al. 2005). We have no simultaneous X-ray and VLA radio observations of NGC 6334B. The available radio fluxes $\sim 0.4 \mathrm{Jy}$ (at $6 \mathrm{~cm}$ ) measured two decades before the Chandra observation are much higher than that expected for a galactic black hole binary in a low-hard state with $\sim$ mCrab flux in $2-10 \mathrm{keV}$ band. The radio flux can be achieved only in a relatively rare high-soft state of a stellar mass black hole binary. Simultaneous X-ray and radio observations of NGC 6334B are needed to finally establish its nature.

The broadband spectral characteristics of NGC 6334B given above are consistent with that observed in obscured accreting sources associated with AGNs (see e.g. 


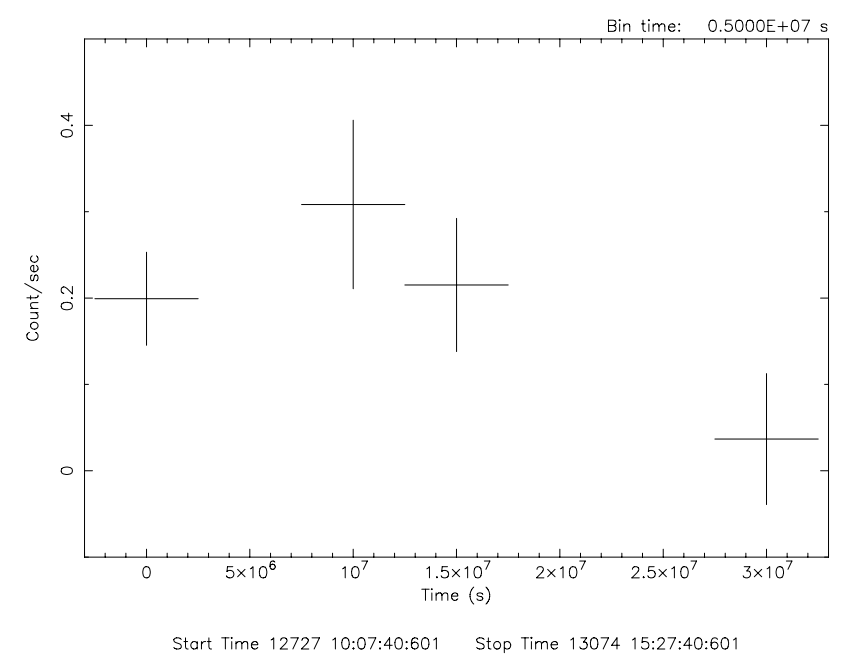

Fig. 6. The 20-40 keV ISGRI lightcurve of NGC 6334B. The bounding dates are in the Truncated Julian Days (TJD) defined as $\mathrm{TJD}=\mathrm{JD}-2440000.5$.

Fabian 2004). The absorbing column density of NGC 6334B $N_{\mathrm{H}} \sim 10^{23} \mathrm{~cm}^{-2}$ is substantially higher than typical values for the NGC 6334 field, where $N_{\mathrm{H}} \lesssim 3 \times 10^{22} \mathrm{~cm}^{-2}$.

Redshifted Fe line complexes are often observed in accreting AGN sources. A dedicated XMM-Newton observation of NGC 6334B can be used to search for the spectral feature and thus to constrain the source redshift. An XMM-Newton observation would also yield a significantly improved X-ray spectra for NGC 6334A and the HEE region above $7 \mathrm{keV}$.

\subsection{Nonthermal emission of the SFR}

SF complexes are expected to have a number of high energy emission sources. Young massive stars with stellar winds, SNRs interacting with molecular clouds, young stellar objects with magnetic activity, jets and Herbig-Haro outflows are among the most plausible candidates. A number of bubbles and shells of different sizes are apparent in the multiwavelength images in Fig. 1 and some of these are identified with winds from young massive stars (e.g. Carral et al. 2002). Moreover, the variability of a powerful FIR source (McBreen et al. 1979) in a close proximity to the ISGRI excess makes it reasonable to consider a buried SNR as a plausible scenario for the HEE. All these objects have to be considered as candidates for the observed hard nonthermal emission.

The observed ISGRI spectrum below $100 \mathrm{keV}$ is well fitted by a hard power law of photon index $\approx 1$. We also obtained a $3 \sigma$ upper limit for the $67.9 \mathrm{keV}$ and $78.4 \mathrm{keV}^{44} \mathrm{Ti}$ lines of $2.1 \times$ $10^{-5} \mathrm{ph} \mathrm{cm}^{-2} \mathrm{~s}^{-1}$. This implies a maximal ${ }^{44} \mathrm{Ti}$ yield of $2.3 \times$ $10^{-6} M_{\odot}$ which is much lower than predicted by most of the current core-collapse SN models for a SN of age $<100$ years (e.g. Woosley \& Weaver 1995).

The powerful (of $L_{\mathrm{b}} \sim 2.5 \times 10^{5} L_{\odot}$ bolometric luminosity) FIR bipolar source with a high velocity and a high luminosity $\mathrm{H}_{2} \mathrm{O}$ maser, some Herbig-Haro objects (e.g. Loughran et al. 1986), and a well defined $\sim 15^{\prime \prime}$ shell that may be an O7.5 star are located in the region (Carral et al. 2002). The VLA radio

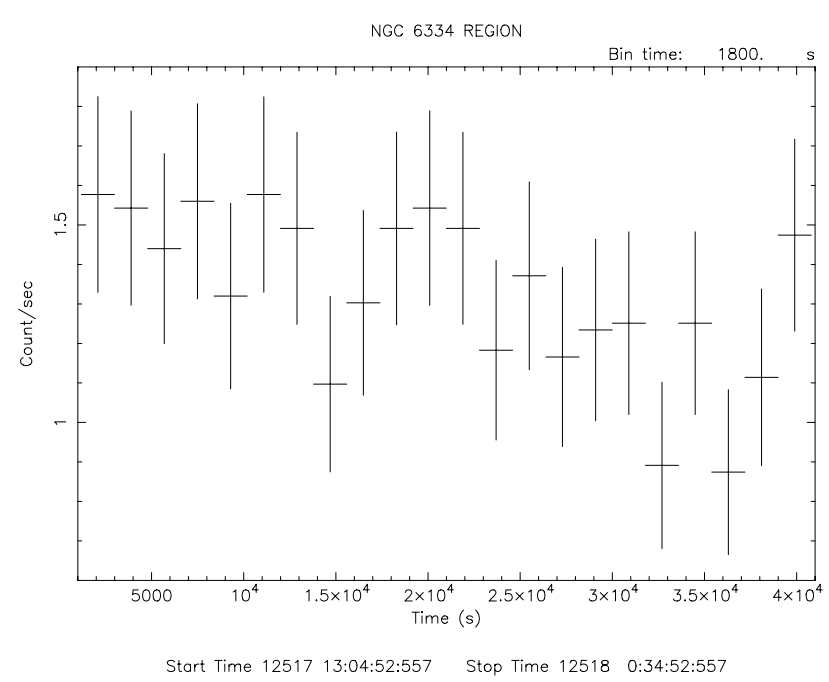

Fig. 7. The 0.8-8.0 keV Chandra light curve of NGC 6334B. The bounding dates are in the Truncated Julian Days (TJD) defined as $\mathrm{TJD}=\mathrm{JD}-2440000.5$.

shell is indicated as a parallelogram frame in the Chandra image in Fig. 4. The mechanical luminosity of the O7.5 star wind was estimated by Carral et al. (2002) to be $L_{\mathrm{w}} \sim 10^{37} \mathrm{erg} \mathrm{s}^{-1}$ i.e. a few percent of $L_{\mathrm{b}}$. If the bolometric luminosity of the source was initially released as a mechanical luminosity of supersonic outflows from young (proto)stellar source population and then converted into the FIR emission then the hard X-ray emission can be produced by bremsstrahlung emission of fast electrons (of energies below $\mathrm{MeV}$ regime) accelerated in the outflows. The efficiency of X-ray bremsstrahlung emission is relatively low due to the Coulomb losses of fast particles, providing powerful IR extended emission from the dense medium. The conversion efficiency of the bremsstrahlung emission is $\eta \sim 10^{-5}$ in an ambient medium [of solar abundance] for the energy band below $\mathrm{MeV}$. One may conclude that hard X-ray emission of $L_{\mathrm{x}} \lesssim 10^{34} \mathrm{erg} \mathrm{s}^{-1}$ can still be produced by the $\mathrm{MeV}$ electrons. A model of electron acceleration by MHD shock waves with velocities about $100 \mathrm{~km} \mathrm{~s}^{-1}$, that are typical for sub-parsec scale size outflows in a dense ambient medium with $n_{\mathrm{a}} \gtrsim 10^{4} \mathrm{~cm}^{-3}$, was made by Bykov et al. (2000). They considered the case of a supersonic flow produced by a supernova shock in a dense clump of a molecular cloud, but the model can be applied to a MHD shock of a similar size in a dense medium produced by any other supersonic outflow, e.g. by a fast wind in the dense medium. A distinctive feature of the model is a very flat X-ray spectrum of photon index $\Gamma \sim 1$. An energetically more efficient model of the hard X-ray emission with the conversion efficiency $\eta \gtrsim 10^{-3}$ could be realized in case of synchrotron emission of $\mathrm{TeV}$ regime electrons accelerated by fast $\left(\gtrsim 1000 \mathrm{~km} \mathrm{~s}^{-1}\right.$ ) shock waves in colliding winds.

Given the high significance of the Chandra detection of both AGN and HEE sources it is also possible that both are contributing to the observed hard X-ray emission. Future high resolution observations with Simbol-X (Ferrando et al. 2004) or Gamma Ray Imager could help to resolve the issue. 


\section{Conclusions}

1. We detected a hard X-ray emission source in the galactic star-forming region NGC 6334 with the $J E M-X$ and IBIS/ISGRI telescopes aboard the International GammaRay Astrophysics Laboratory INTEGRAL. The source has a nonthermal spectrum at least up to $100 \mathrm{keV}$.

2. From the multiwavelength analysis of the complex NGC 6334 region we concluded that the source may be associated both with the background, likely extragalactic, radio source NGC 6334B projected onto the NGC 6334 SFR, and with an extended HII region, associated with bright IR source and a radio shell NGC 6334A.

Acknowledgements. We thank the anonymous referee for constructive comments. The work was partially supported by RFBR grants 03-02-17433, 04-02-16595, 04-02-16042, 03-07-90200, RAS program, Russian Leading Scientific Schools grant 1115.2003.2, and by the ESA. J.C.L., G.R. and J.P.S. acknowledge support through the XMM-INTEGRAL PRODEX project, the Belgian FNRS, and IAP contract P5/36. Support from the International Space Science Institute (Bern) through the international teams program is gratefully acknowledged.

\section{References}

Bykov, A. M. 2001, Space Sci. Rev., 99, 317

Bykov, A. M., Chevalier, R. A., Ellison, D. C., \& Uvarov, Yu. A. 2000, ApJ, 538, 203
Carral, P., Kurtz, S. E., Rodriguez, L. F., et al. 2002, AJ, 123, 2574

Courvoisier, T. J.-L., Walter, R., Beckmann, V., et al. 2003, A\&A, 411, L53

Ezoe, Y., Kokubun, M., \& Makishima, K. 2005, in Star Formation in the Era of Three Great Observatories, 49

Fabian, A. C. 2004, in Coevolution of Black Holes and Galaxies, ed. L. Ho (Cambridge University Press), 447

Fender, R. P. 2005, in Compact Stellar X-ray Sources, ed. W. H. G. Lewin, \& M. van der Klis (Cambridge University Press) [arXiv: astro-ph/0303339]

Ferrando, Ph., Arnaud, M., Cordier, B., et al. 2004, Proc. SPIE, 5168, 65

Kraemer, K. E., \& Jackson, J. M. 1999, ApJS, 124, 439

Lebrun, F., Leray, J. P., Lavocat, P., et al. 2003, A\&A, 411, L141

Loughran, L., McBreen, B., Fazio, G. G., et al. 1986, ApJ, 303, 629

Lund, N., Budtz-Joergensen, C., Westergaard, N. J., et al. 2003, A\&A, 411, L231

Matsuzaki, K., Sekimoto, Y., Kamae, T., et al. 1999, Astron. Nachr., 320(4), 323

McBreen, B., Fazio, G. G., Stier, M., \& Wright, E. L. 1979, ApJ, 232, L183

Moran, J. M., Rodriguez, L. F., Greene, B., et al. 1990, ApJ, 348, 147

Muno, M. P., Belloni, T., Dhawan, V., et al. 2005, ApJ, 626, 1020

Neckel, T. 1978, A\&A, 69, 51

Sarma, A. P., Troland, P. H., Roberts, D. A., et al. 2000, ApJ, 533, 271

Sekimoto, Y., Matsuzaki, K., Kamae, T., et al. 2000, PASJ, 52, L31

Ubertini, P., Lebrun, F., Di Cocco, G., et al. 2003, A\&A, 411, L131

Winkler, C., Courvoisier, T., Di Cocco, G., et al. 2003, A\&A, 411, L1

Woosley, S. E., \& Weaver, T. A. 1995, ApJS, 101, 181 